\title{
Skin Cyst
}

National Cancer Institute

\section{Source}

National Cancer Institute. Skin Cyst. NCI Thesaurus. Code C3884.

A cystic lesion located in the skin. 